\title{
High-frequency waves in solar and stellar coronae
}

\author{
V. G. Ledenev ${ }^{1}$, V. V. Tirsky ${ }^{2}$, and V. M. Tomozov ${ }^{1}$ \\ 1 Institute of Solar-Terrestrial Physics, Russian Academy of Sciences, PO Box 4026, Irkutsk, Russia \\ e-mail: leden@iszf.irk.ru \\ 2 Institute of Laser Physics, Russian Academy of Sciences, Irkutsk Branch, Russia \\ Received 3 July 2006 / Accepted 15 November 2006
}

\section{ABSTRACT}

\begin{abstract}
On the basis of a numerical solution of dispersion equation we analyze characteristics of low-damping high-frequency waves in hot magnetized solar and stellar coronal plasmas in conditions when the electron gyrofrequency is equal or higher than the electron plasma frequency. It is shown that branches that correspond to Z-mode and ordinary waves approach each other when the magnetic field increases and become practically indistinguishable in a broad region of frequencies at all angles between the wave vector and the magnetic field. At angles between the wave vector and the magnetic field close to $90^{\circ}$, a wave branch with an anomalous dispersion may occur. On the basis of the obtained results we suggest a new interpretation of such events in solar and stellar radio emission as broadband pulsations and spikes.
\end{abstract}

Key words. plasmas - turbulence - radiation mechanisms: non-thermal - Sun: flares - Sun: radio radiation - stars: flare

\section{Introduction}

It has been widely accepted that numerous flare processes with energy release take place in solar and stellar coronal plasma on different scales both in time and space (Altyntsev et al. 1982; Somov 1992; Sakai \& de Jager 1996; Gershberg \& Pikelner 1972; Gershberg 2002; Stepanov 2003). It is believed that flares originate as a result of magnetic energy release. This energy has previously accumulated in an active region. In turn, it means that plasma parameters of stellar and solar coronae in the flare region differ from parameters of quiet coronae through the stronger magnetic field. At the present time flare parameters measurements in stellar and solar coronae is an unsettled problem, and measuring magnetic field is the most difficult one. Some progress has been achieved in determination of the magnetic field in the solar corona. In a considerable number of papers, the magnetic field in a flare region is evaluated from parameters of radio emission bursts of the Sun (Dulk \& McLean 1977; Mann et al. 1987; Ledenev \& Messerotti 1999; Ledenev et al. 2001; Vrŝnak et al. 2002). But these magnetic field estimations essentially depend on an accepted model of radio emission generation, so they give results that differ by an order of magnitude. Thus, conclusions about the magnetic field value and its time variations can be made from theoretical calculations in the framework of accepted flare models (Altyntsev et al. 1982; Somov 1992; Sakai \& de Jager 1996; Priest \& Forbes 2000).

Two-dimensional models of extended (plane) current sheets (Somov 1992; Priest \& Forbes 2000) and magnetic loop interaction (merging) models (Sakai \& de Jager 1996) are the most elaborated ones. Calculations show that the magnetic field may increase by an order of magnitude during formation of extended current sheets (Anderson \& Priest 1993); and during magnetic loop merging, the axial component of the field may increase by more than two orders of magnitude (Chargeishvili et al. 1993). This means that, in a build-up flare process in a local coronal region, the ratio of the electron gyrofrequency $\omega_{\mathrm{He}}$ to the plasma frequency $\omega_{\text {pe }}$ may increase substantially. The value of this ratio is essential for determining plasma eigen oscillation spectra in the magnetic field and, consequently, for determining solar coronal parameters in the flare region. Magnetic field estimations give values of the ratio $\omega_{\mathrm{He}} / \omega_{\mathrm{pe}}<1$ (Ledenev et al. 2001; Vrŝnak et al. 2002). However, it is very likely that the magnetic field grows to such values that this ratio becomes higher than unity in the course of a flare during the short period of about one second or less.

It is very important to take the finite plasma temperature into account for determining eigen oscillations spectra in the magnetized plasma. As shown earlier for $\omega_{\mathrm{He}} / \omega_{\mathrm{pe}}<1$, spectra of hot magnetized plasma oscillations differ from spectra of a cold plasma in such a way that in the region of parameter values $k v_{\mathrm{Te}} / \omega_{\mathrm{He}} \sim 1$ ( $k$ is the wave number and $v_{\mathrm{Te}}$ is the thermal velocity), the oscillation frequency grows when the refractive index $n$ increases (Ledenev et al. 2006). This means that the frequency range of Langmuir waves, which may be generated at a given level of the solar corona, enlarges substantially (up to $30-40 \%$ ). It will be shown that, in the range of values $\omega_{\mathrm{He}} / \omega_{\mathrm{pe}}>1$, the spectrum of eigen oscillations of the hot magnetized plasma displays some features that may determine a fine structure of solar radio emission. In particular, at $\omega_{\mathrm{He}} / \omega_{\mathrm{pe}} \sim 1 \mathrm{a}$ branch with negative dispersion appears in the longitudinal wave spectrum; i.e. waves can propagate whose frequency decreases with the growth of the wave number. At $\left(\omega_{\mathrm{He}} / \omega_{\mathrm{pe}}\right)^{2} \gg 1$, the branch corresponding to Z-mode (at small angles between the wave vector and the magnetic field, the branch corresponding to whistler mode) approaches the ordinary wave branch, and these waves become indistinguishable when the value $\left(\omega_{\mathrm{He}} / \omega_{\mathrm{pe}}\right)^{2}$ increases. These characteristics of the eigen oscillation spectra of the coronal plasma allow us to explain the origin of broad-band pulsations that are observed in solar radio emission on the basis of magnetic field pulsations in the reconnection region and to suggest a new mechanism for the generation of narrowband short-living bursts (spikes).

In the present paper we investigate different types of highfrequency waves in the high-temperature magnetized plasma for values of the parameter $\omega_{\mathrm{He}} / \omega_{\mathrm{pe}} \geq 1$. In Sect. 2 the 
dispersion equation describing high-frequency waves in the high-temperature magnetized plasma is represented in its most general form. A numerical solution of the dispersion equation is described in detail, and high-frequency wave characteristics are represented taking into account their propagation at different angles to the magnetic field. In Sect. 3 on the basis of the results for high-frequency waves in the hot coronal plasma, we provide a qualitative interpretation of some features of solar radio emission such as broadband pulsations and spikes. Results of this work are reported and discussed in the conclusion.

\section{Spectra of high-frequency waves in a high-temperature magnetized plasma}

In spite of a considerable amount of papers and books focused on the study of eigen oscillations in the high-temperature magnetized plasma (see, e.g., Baldwin et al. 1969; Ginnzburg 1970; Akhiezer et al. 1975; Ginzburg \& Rukhadze 1975), this problem has not been completely resolved at the present time. The point is that it is necessary to use the kinetic approach for taking the finite temperature effect into account, e.g. for considering the influence of the velocity distribution function of particles on plasma dispersion characteristics. The effect of finite electron gyroradius (effect of strong spatial dispersion) is a principal effect, which influences plasma wave properties, and it appears when the wavelength becomes comparable to the electron gyroradius.

Characteristics of high-frequency waves in the hightemperature plasma with parameters corresponding to the magnetospheric plasma were studied by Andre (1985). Recently Ledenev et al. (2006) investigated properties of such waves in a plasma with parameters corresponding to the solar corona in the frequency range $\omega>\omega_{\mathrm{He}}$ ( $\omega$ is the wave frequency, $\omega_{\mathrm{He}}$-electron gyrofrequency) taking the wave damping into account. This study was made on the basis of a numerical solution of the dispersion equation for the high-temperature magnetized plasma (Akhiezer et al. 1975; Ginzburg \& Rukhadze 1975)

$a n^{4}+b n^{2}+c_{0}=0$

where

$$
\begin{aligned}
a= & \left(k_{\perp}^{2} / k^{2}\right) \varepsilon_{x x}+\left(k_{z}^{2} / k^{2}\right) \varepsilon_{z z}+2\left(k_{\perp} k_{z} / k^{2}\right) \varepsilon_{x z}, \\
b= & -\varepsilon_{x x} \varepsilon_{z z}+\varepsilon_{x z}^{2}-\left(k_{z} / k\right)^{2}\left(\varepsilon_{y y} \varepsilon_{z z}+\varepsilon_{y z}^{2}\right)-\left(k_{\perp} / k\right)^{2} \\
& \times\left(\varepsilon_{x x} \varepsilon_{y y}+\varepsilon_{x y}^{2}\right)+2\left(k_{\perp} k_{z} / k^{2}\right)\left(\varepsilon_{x y} \varepsilon_{y z}-\varepsilon_{x z} \varepsilon_{y y}\right), \\
c_{0}= & \varepsilon_{z z}\left(\varepsilon_{x x} \varepsilon_{y y}+\varepsilon_{x y}^{2}\right)+\varepsilon_{x x} \varepsilon_{y z}^{2}-\varepsilon_{y y} \varepsilon_{x z}^{2}+2 \varepsilon_{y z} \varepsilon_{x z} \varepsilon_{x y} .
\end{aligned}
$$

Here, $n=k c / \omega, \omega$ is the complex frequency, $k$ the wave number, $k_{z}$ the longitudinal (with respect to the magnetic field) wave vector component, $k_{\perp}$ the transverse wave vector component, and $\varepsilon_{i j}$ stands for the components of the dielectric tensor.

Dispersion properties of waves change in the frequency region near the electron plasma frequency and in the regions where the refractive index $n \leq 1$ and $n \gg 1$. In the region $n \leq 1$, longitudinal waves turn to Z-mode, and in the region $n \gg 1$ the frequency of longitudinal waves increases with growth of n. At angles close to $90^{\circ}$ and for $n \gg 1$, longitudinal waves turn into waves with anomalous dispersion, i.e. waves whose frequency decreases as the wave number grows.

In this paper spectra of eigen oscillations of the hightemperature magnetized plasma are investigated in the frequency range $\omega \leq \omega_{\mathrm{He}}$ for values of the parameter $\eta=\omega_{\mathrm{pe}} / \omega_{\mathrm{He}} \leq 1$. These parameter values may be realized at definite conditions in the solar corona and also in stellar coronae. Figures 1a-f represent solutions of Eq. (1) (by formulas A.1 from the paper by Ledenev et al. 2006) as the dependence of the refractive in$\operatorname{dex} n$ on the frequency. The solutions of Eq. (1) were found by a graphic method, with a step of calculation of $0.01 \omega_{\mathrm{He}}$. Calculations are made for (a) $\eta=\omega_{\mathrm{pe}} / \omega_{\mathrm{He}}=1$, the angle between the wave vector and the magnetic field (radians) $\theta=0$, (b) $\eta=1, \theta=1$, (c) $\eta=1, \theta=1.5$, (d) $\eta=0.25, \theta=0$, (e) $\eta=0.25, \theta=1$, and (f) $\eta=0.25, \theta=1.5 ;\left(v_{\mathrm{Te}} / c\right)^{2}=2 \times 10^{-4}$. The plasma temperature corresponds to the solar corona temperature.

As can be seen from Figs. 1a-f, the character of the dispersion curves for extraordinary (branch 1) and ordinary (branch 2) waves does not change in comparison with the cold plasma. Cutoff frequencies at $n=0$ are determined with the same relations $\omega_{\mathrm{c}} / \omega_{\mathrm{pe}}=(1 / 2)\left\{ \pm\left(\omega_{\mathrm{He}} / \omega_{\mathrm{pe}}\right)+\left[\left(\omega_{\mathrm{He}} / \omega_{\mathrm{pe}}\right)^{2}+4\right]^{1 / 2}\right\}$ (Ginzburg 1970), where the upper sign refers to extraordinary and the lower one to ordinary waves. At $\eta=1$ we have $\omega_{\mathrm{cx}} / \omega_{\mathrm{pe}} \approx 1.62$ for extraordinary waves and $\omega_{\mathrm{co}} / \omega_{\mathrm{pe}} \approx 0.62$ for ordinary waves when they propagate along the magnetic field. For $\eta=0.25$, we have $\omega_{\mathrm{c} x} / \omega_{\mathrm{He}} \approx 1.06$ and $\omega_{\mathrm{co}} / \omega_{\mathrm{He}} \approx 0.06$, respectively. For wave propagation at an angle to the magnetic field, the existence range of ordinary waves is limited by the electron plasma frequency. All these values correspond to our calculations.

The whistler branch is shown in Figs. 1a and 1d (branch 5). It is located in the frequency range from $\sim 0.1 \omega_{\mathrm{He}}$ to $\omega_{\mathrm{He}}$. As can be seen from Fig. 1a, the intersection of branches 2 and 3 occurs for $\eta=1$ in the case of wave propagation along the magnetic field $(\theta=0)$, and a transition takes place from one wave type into another (linear wave interaction). The intersection of branches is absent when waves propagate at an angle to the magnetic field (Fig. 1b), when longitudinal waves (branch 3) pass into Z-mode (branch 4), and whistlers pass into fast magnetosonic waves (branch 6 in Fig. 1b). When the angle $\theta$ grows to $90^{\circ}$, the frequency of longitudinal waves increases and approaches the upper hybrid frequency $\omega_{\mathrm{g}}=\left(\omega_{\mathrm{pe}}^{2}+\omega_{\mathrm{He}}^{2}\right)^{1 / 2} \approx 1.4 \omega_{\mathrm{pe}}$ (Fig. 1c).

The intersection of the longitudinal wave branch (branch 3 in Fig. 1d) with whistler's dispersion curves (branch 5) and with ordinary waves (branch 2 ) at $\eta=0.25$ occurs when the waves propagate along the magnetic field. For $\eta=0.25$ the longitudinal wave branch shifts to the range of lower frequencies when the angle $\theta$ grows, it is identified as the fast magnetosonic wave (branch 6 in Fig. 1e), and whistlers pass into Z-mode (branch 4).

An asymptotical value of the fast magnetosonic wave frequency at $n \gg 1$ is equal to $\omega_{\infty}=\omega_{\mathrm{pe}} \omega_{\mathrm{He}} \cos \theta /\left(\omega_{\mathrm{pe}}^{2}+\omega_{\mathrm{He}}^{2}\right)^{1 / 2}$ (Akhiezer et al. 1975). In the case for $\eta=1$ and $\theta=1$ radian, we have $\omega_{\infty} / \omega_{\text {pe }} \approx 0.38$ at $n \sim 10$ (Fig. 1b) (at $n>10$ the increase in the frequency with growth of $n$ occurs due to spatial dispersion). At $\eta=0.25$ and $\theta=1$ radian, we have $\omega_{\infty} / \omega_{\text {pe }} \approx 0.14$ (Fig. 1e).

As can be seen from Fig. 1e, at $\eta=0.25$ the branch of longitudinal waves is absent at intermediate angles seemingly because of cyclotron damping, and it appears again at angles $\theta$ close to $90^{\circ}$. In this case its frequency is close to the upper hybrid frequency (Fig. 1f). At $\eta=1$ the longitudinal waves (branch 3) exist at all angles $\theta$. In the region of $n>10$, the frequency of these waves increases with the growth of $n$, but waves with anomalous dispersion appear close to $\theta=90^{\circ}$, whose frequency falls with the growth in the refractive index (branch 7 in Fig. 1c). Waves with anomalous dispersion are absent (Fig. 1f) for a stronger magnetic field $(\eta=0.25)$.

It follows from our calculations (Figs. 1d-f) that branches 2 and 4 (at small angles $\theta$ branches 4 and 5) approach with the magnetic field enhancement (parameter $\eta$ decreases) in the 


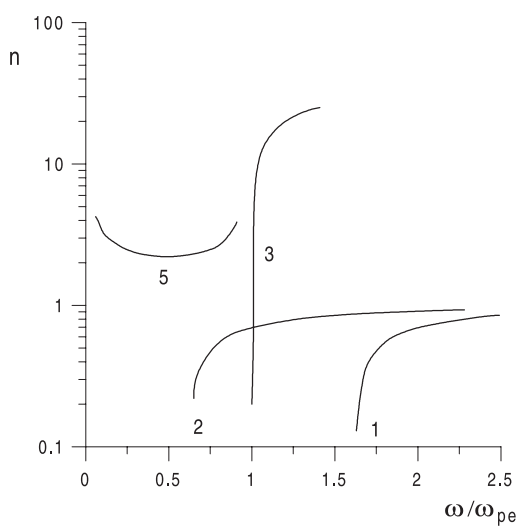

b
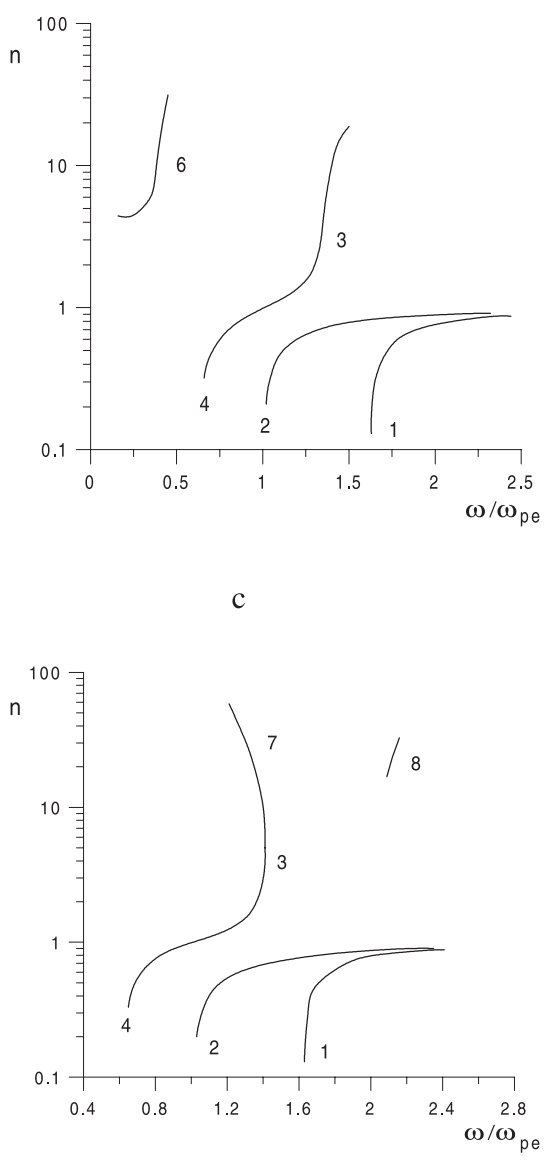

d

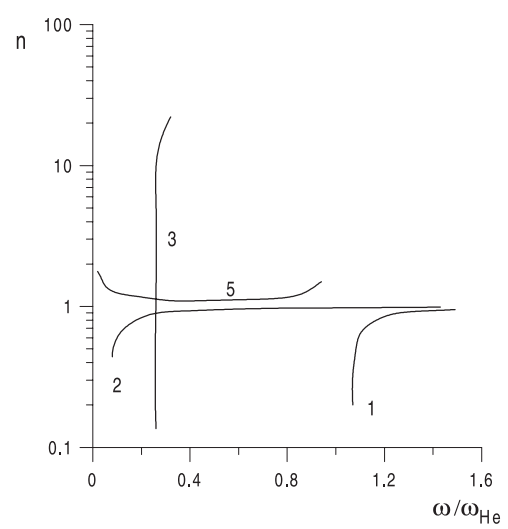

e

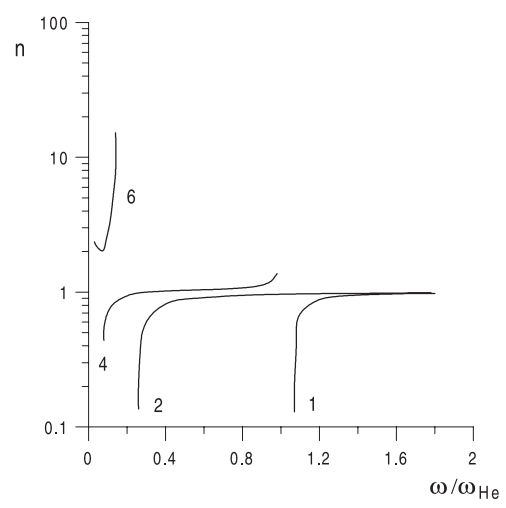

f

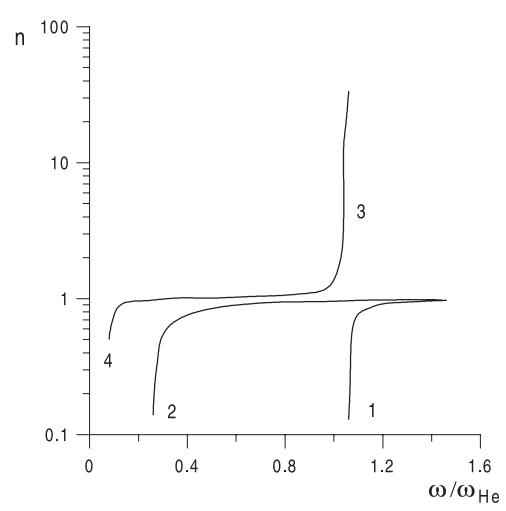

Fig. 1. Frequency dependence of the refractive index $n$ for $\omega_{\mathrm{pe}} / \omega_{\mathrm{He}}=1$, the angle between the wave vector and the magnetic field $\theta=0.0$ (panel a)), $\theta=1.0$ radian (panel b)), $\theta=1.5$ radian (panel c)), and for $\omega_{\mathrm{pe}} / \omega_{\mathrm{He}}=0.25$, $\theta=0.0$ (panel d)), $\theta=1.0$ radian (panel e)), $\theta=1.5$ radian (panel f)). Branches 1,2 , and 3 correspond to the extraordinary, ordinary, and longitudinal modes, respectively. Branch 4 corresponds to the Z-mode, curve 5 corresponds to whistlers, curve 6 corresponds to fast magnetosonic waves, branch 7 corresponds to the waves with anomalous dispersion, branch 8 is the manifestation of the second harmonic of Bernstein mode. $v_{\mathrm{Te}}^{2} / c^{2}=2 \times 10^{-4}$. whole of the angle range from $0^{\circ}$ to $90^{\circ}$, and for $\eta=0.25$ the refractive indices are so very close that these waves are practically indistinguishable in a broad range of frequencies $\left(\Delta \omega / \omega_{\mathrm{He}} \sim 0.5\right)$. Besides, at $\eta=1$ and $\theta$ close to $90^{\circ}$, the relatively low-damping branch 8 (Fig. 1c) occurs in narrow ranges of frequencies and values of the refractive index. It corresponds to the second harmonic of the Bernstein mode. When waves propagate at an angle to a sufficiently strong magnetic field, an interval of refractive index values exists where high-frequency waves do not propagate. These values of refractive index are in the range $1.4<n<2$ (Fig. 1e) for $\eta=0.25$.

It should be noted that the plasma temperature variation does not change the character of dispersion dependencies. Maximal values of the refractive index of low-damping longitudinal waves change mainly with the temperature variation. These changes are inversely proportional to electron thermal velocity.

\section{Two radio emission features of the Sun and stars}

\subsection{Broadband pulsations}

Broadband quasi-periodic pulsations are one of the most enigmatic events in the radio emission of the Sun and stars (Bastian et al. 1990; Aschwanden et al. 1999; Stepanov 2003; Aschwanden 2004). A considerable number of models for this event are based on magnetic loop MHD oscillations (Roberts et al. 1983; Nakariakov et al. 2003), nonlinear regimes of kinetic instabilities (Aschwanden 1990) and oscillatory regimes 


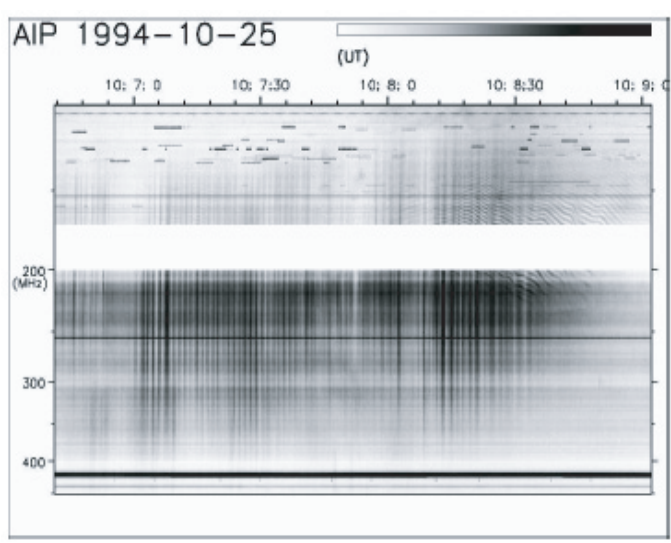

Fig. 2. Broadband pulsations in solar radio emission observed with the Astrophysical Institute Potsdam spectrograph.

of magnetic reconnection (Kliem et al. 2000). In our opinion the magnetic-reconnection process is the most promising one for the origin of broadband pulsations because it is followed by fast changes in plasma parameters by significant magnitude with simultaneous acceleration of particles, and it often shows a quasiperiodic (pulsating) behavior. Results of numerical modelling (Anderson \& Priest 1993; Chargeishvili et al. 1993) and laboratory experiments (Altyntsev et al. 1977; Frank \& Bogdanov 2001) show that, in the reconnection region during the flare process, the magnetic field value may episodically exceed its value significantly before the flare begins. If plasma parameters before the flare are such that $\omega_{\mathrm{He}} / \omega_{\mathrm{pe}}<1$, then in a build-up flare process (free magnetic field energy increase) this ratio may change into an inverse one, i.e. $\omega_{\mathrm{He}} / \omega_{\mathrm{pe}}>1$. If the flare process has a pulsating character, this ratio also changes intermittently. When the inequality $\omega_{\mathrm{He}} / \omega_{\mathrm{pe}}>1$ is fulfilled, dispersion characteristics of eigen oscillations change (Figs. 1a-f). In particular, waves can propagate in a broad range of frequencies beneath $\omega_{\mathrm{He}}$ with the refractive index, which is presumably constant and close to unity (Figs. 1d-f). It means that the same group of energetic electrons can excite oscillations in a broad band of frequencies. If the reconnection process has a pulsating character, radio emission will be generated as a sequence of broadband pulses (Fig. 2).

Our calculations show that for $\left(\omega_{\mathrm{He}} / \omega_{\mathrm{pe}}\right)^{2} \gg 1$ the refractive index of waves corresponding to Z-mode (at small angles between the wave vector and the magnetic field corresponding to whistlers) is close to unity in a broad range of frequencies lower than $\omega_{\mathrm{He}}\left(\Delta \omega \sim \omega_{\mathrm{He}}\right)$. The frequency of waves is determined by the dispersion relation $n^{2}=\varepsilon$ when they propagate along the magnetic field (Mikhailovsky 1974), where $\varepsilon$ is dielectric permittivity or

$(k c)^{2} / \omega^{2}=1-\omega_{\mathrm{pe}}^{2} / \omega\left(\omega-\omega_{\mathrm{He}}\right)$.

At $\left(\omega_{\mathrm{He}} / \omega_{\mathrm{pe}}\right)^{2} \gg 1$, the solution of this equation leads to two approximate values of frequency $\omega_{1} \approx k c-\omega_{\mathrm{pe}}^{2} / 2\left(k c+\omega_{\mathrm{He}}\right) \approx k c$ and $\omega_{2} \approx \omega_{\mathrm{He}}$. The first value is the frequency of whistlers and the second one refers to the longitudinal wave branch, which is strongly damped when the waves propagate at small angles to the magnetic field.
The growth rate of whistlers in the case of wave propagation along the magnetic field $\left(\omega \approx k_{z} c\right)$ has the form (Mikhailovsky 1974)

$$
\begin{aligned}
\gamma= & -\operatorname{Im} \varepsilon /(\partial \operatorname{Re} \varepsilon / \partial \omega)=\frac{c\left(k_{z} c-\omega_{\mathrm{He}}\right)^{2}}{2 n\left(2 k_{z} c-\omega_{\mathrm{He}}\right)} \int v_{\perp}^{3} \mathrm{~d} v_{\perp} \\
& \times\left[\frac{\partial F}{v_{\perp} \partial v_{\perp}}+\frac{1}{c}\left(\frac{\partial F}{\partial v_{\|}}-v_{\|} \frac{\partial F}{v_{\perp} \partial v_{\perp}}\right)\right]_{v_{\|}=v_{\mathrm{res}}},
\end{aligned}
$$

where $F$ is the energetic electron distribution function, $k_{z}$ the longitudinal wave vector component in relation to the magnetic field, $n$ plasma density, and $v_{\text {res }} \approx c-\omega_{\mathrm{He}} / k_{z}$.

As is known (Litvinenko 2002), acceleration of electrons occurs mainly along the magnetic field during the reconnection in the current sheet with a weak shear of the magnetic field. The distribution function of accelerated electrons has a power-law character (Litvinenko 1996). In laboratory plasma experiments, an extended and almost flat (up to a certain limit) tail of accelerated electrons is observed (Altyntsev et al. 1977). The conclusion follows from this substance that the $\partial F / \partial v_{\|}$in Eq. (2) may be neglected. In the case of the assumption of Maxwell distribution over transverse velocities, the expression for the growth rate has the form:

$$
\begin{aligned}
\gamma & \approx \frac{c\left(k_{z} c-\omega_{\mathrm{He}}\right)^{2}}{\pi n\left(\Delta v_{\|}\right)\left(2 k_{z} c-\omega_{\mathrm{He}}\right)}\left(1-\frac{v_{\|}}{c}\right) F\left(v_{\|}\right) \\
& \approx \frac{\omega_{\mathrm{He}}\left(k_{z} c-\omega_{\mathrm{He}}\right)^{2}}{\pi n\left(\Delta v_{\|}\right)\left(2 k_{z} c-\omega_{\mathrm{He}}\right) k_{z}} F\left(v_{\mathrm{res}}\right) .
\end{aligned}
$$

As is seen from expression (3), whistlers are excited in the frequency range $\omega_{\mathrm{He}} / 2<\omega_{1} \approx k_{z} c<\omega_{\mathrm{He}}$. Since the waves have practically the same phase velocities, their generation occurs simultaneously in the whole frequency band as observed (see Fig. 2). The restriction on the frequency from the bottom is determined by the condition that the derivative $\mathrm{d} n / \mathrm{d} \omega$ changes the sign at $\omega \approx \omega_{\mathrm{He}} / 2$ and, consequently, the derivative $\mathrm{d} \varepsilon / \mathrm{d} \omega$ changes the sign too, as follows from the dispersion equation. From this we obtain estimations of the magnetic field corresponding to the time of the emission. The cyclotron frequency corresponds to the high-frequency radiation boundary and $\omega_{\mathrm{He}} / 2$ corresponds to the low-frequency boundary; i.e. frequencies corresponding to both upper and lower boundaries of the radiation band differ by a factor two. However, another wave type (Z-mode) is excited at the angle to the magnetic field. The dispersion dependence of these waves, as is seen from Figs. 1e and $\mathrm{f}$, decreases without the derivative sign changing when the frequency decreases, and the radiation band enlarges towards lower frequencies as long as the refractive index is larger than unity.

The whistler's and Z-mode's transition into transverse electromagnetic waves occurs due to linear conversion of one wave into another (Zheleznyakov 1970), because, as one can see from Figs. 1d,f, the dispersion plots of these waves practically merge with the plot of ordinary electromagnetic waves when the magnetic field is strong enough.

As Kliem et al. (2000) show, the reconnection process in an extended current sheet may represent oscillations of plasma parameters with a characteristic time of about one second. It may explain an origin of broadband pulsations in solar radio emission. However, this model, in our opinion, does not decisively explain the broad band of radiation and the absence of a frequency drift in observed pulsations. If we take into account that the magnetic field in the reconnection process may be essentially 
intensified, then the broadband pulsations and the absence of the frequency drift are explained naturally.

The proposed model of broadband pulsations can be applied completely to the radio emission pulsations of flare stars (Bastian et al. 1990; Abada-Simon et al. 1997) since the magnetic field on their surface is stronger, and the flare energy may be $10^{3}$ times higher than the solar flare energy.

\subsection{Spikes}

Short-term (its duration is less than one second) narrow-band $(\Delta \omega / \omega \sim 1 \%)$ bursts of solar radio emission are known as spikes (Benz 1986; Fleishman \& Melnikov 1998). It is assumed that these bursts are generated by plasma or maser mechanisms (Fleishman \& Melnikov 1998). Conclusive evidence has not yet been found in favor of any mechanism. Our calculations of highfrequency wave spectra in the magnetized plasma allow us to propose a generation mechanism based on features of the plasma eigen oscillation spectrum, which was not known previously. It allows us to determine plasma parameters in the magnetic reconnection region more precisely.

If the reconnection process occurs in a small region, which is at a high coronal level, it manifests itself mainly in the form of radio emission bursts in decimeter and meter ranges. Since plasma parameters in the reconnection region essentially change within a small time scale, then in some moments of time in this region the electron cyclotron frequency may be equal to the electron plasma frequency. As seen from Fig. 1c, in this case the well-defined branch with anomalous dispersion (branch 7) appears in the spectrum of longitudinal waves, which propagate at angles to the magnetic field close to $90^{\circ}$. At the point of transition from waves with normal dispersion (branch 3) to waves with anomalous dispersion (branch 7), the frequency derivative of the refraction index equals infinity, i.e. the wave group velocity tends to zero. It means that the longitudinal waves will be accumulated in this region and then radiate as intensive radio emission. We assume that the narrow-band short-time bursts of solar radio emission (spikes) are generated in this way. The narrow band of the radiation may be explained by the fact that the wave frequency changes insignificantly in a broad interval of wave phase velocities; i.e. the wave accumulation proceeds in an extensive range of wave numbers and in a narrow interval of frequencies. In this case the dimension of the radiation region must be essentially smaller than a characteristic scale of the inhomogeneous corona. The small duration of bursts is determined by the circumstance that the equality of cyclotron and plasma frequencies occurs during a short interval of time, because plasma parameters in the reconnection regime change continuously. If the characteristic reconnection time is about one second (Kliem et al. 2000), then the duration of this burst is a fraction of a second. Radiation is generated at the double-frequency of longitudinal waves at their coalescence, i.e. $\omega \approx 2.8 \omega_{\text {pe }}$, because the upper hybrid frequency is $1.4 \omega_{\text {pe }}$ in this case.

Observations (Krucker \& Benz 1994) show that spikes are often observed as large groups in the form of two emission bands with the ratio of frequencies, which does not exceed the value 1.4 in most cases. We assume that such groups are generated when a pulsating regime of the magnetic reconnection exists. In this case radiation corresponding to the higher frequency is generated as a result of longitudinal wave merging at the frequency $\omega \approx 2,8 \omega_{\text {pe }}$, and emission corresponding to the lower frequency is generated by merging longitudinal waves with whistlers when they propagate along the magnetic field (Fig. 1a). The most favorable conditions for the merging process of longitudinal waves and whistlers are realized when cyclotron and plasma frequencies are close. When waves propagate along the magnetic field, parameters of whistlers and longitudinal waves draw together (Fig. 1a) and the merging process is analogous to the coalescence of two longitudinal waves. In this case the radiation frequency is close to the double of the plasma frequency. It should be noted that the frequency ratio 1.4 corresponds to the equality of cyclotron and plasma frequencies. If the cyclotron frequency in the reconnection process stays lower than the plasma frequency, then this ratio will be less than 1.4 as has been observed (Krucker \& Benz 1994).

\section{Conclusions}

Our calculations show that the finite temperature of the magnetized plasma affects its dispersion characteristics essentially. In this paper the plasma in a sufficiently strong magnetic field is investigated (parameter $\eta=\omega_{\mathrm{pe}} / \omega_{\mathrm{He}} \leq 1$ ). Modes, which appear in the frequency band under consideration, are electromagnetic (transverse) waves (ordinary and extraordinary modes), longitudinal waves, whistlers, Z-mode and fast magnetosonic waves. Whistlers propagate at small angles to the magnetic field. When the angle $\theta$ between the wave vector and the magnetic field grows, whistlers transform into fast magnetosonic waves, which propagate in a frequency band below $\omega_{\text {pe }}$. The high-frequency part of whistlers transforms into Z-mode at $\eta<1$ with a growth in angle $\theta$. The most distinct effect of the finite temperature is the appearance of the wave branch with anomalous dispersion at $\eta=1$ and angles $\theta$ close to $90^{\circ}$. When the parameter $\eta$ decreases, branches corresponding to Z-mode and ordinary waves approach, and they become practically indistinguishable in a wide band of frequencies at all angles $\theta$ for sufficiently low values of the parameter $\eta(\eta \leq 0.25)$.

These calculations allow us to explain some features of radio emission of the Sun and stars in a more natural way. The result that whistlers and Z-mode in a sufficiently strong magnetic field are generated simultaneously in the broad band of frequencies allows us to explain the origin of broadband pulsations in radio emission of the Sun and stars. It is assumed that sufficiently strong magnetic fields are realized in the nonstationary (pulsating) regime of the magnetic field reconnection.

The short-time, narrow-band bursts - spikes are explained by plasma wave accumulation in the transition region from waves with normal dispersion to waves with anomalous dispersion, where the wave group velocity approaches zero. This case is realized if waves propagate at large angles to the magnetic field when the plasma frequency is close to the cyclotron frequency.

Acknowledgements. This work was carried out with support of RFBR grant 04-02-39003, Program of basic research RAS N34, and the Scientific School grants 4741.2006.2. We acknowledge H. Aurass (Astrophysical Institute, Potsdam), who kindly provided the spectrographic radio data. The authors express appreciation to the referee for useful corrections that assisted them in improving the paper.

\section{References}

Abada-Simon, M., Lecacheux, A., Aubier, M., \& Bookbinder, J. A. 1997, A\&A, 321,841

Akhiezer, A. I., Akhiezer, I. A., Polovin, R. V., et al. 1975, Plasma Electrodynamics (Oxford: Pergamon Press)

Altyntsev, A. T., Krasov, V. I., \& Tomozov, V. M. 1977, Sol. Phys., 55, 69

Altyntsev, A. T., Banin, V. G., Kuklin, G. V., \& Tomozov, V. M. 1982, Solar Flares (M.: Nauka)

Anderson, C., \& Priest, E. R. 1993, JGR, 98, 19395

Andre, M. 1985, J. Plas. Phys., 33, 1 
Aschwanden, M. J. 1990, A\&AS, 85, 1141

Aschwanden, M. J. 2004, Physics of the Solar Corona. An Introduction (Chichester, UK: Praxis Publishing Ltd., and Berlin: Springer-Verlag)

Aschwanden, M. J., Fletcher, L., Schrijver, C. J., \& Alexander, D. 1999, ApJ, 520,880

Baldvin, D. E., Bernstein, I. B., \& Veenink, M. P. H. 1969, Advances in Plasma Physics, Vols. 3, 4 (N-Y.: Interscience Publ.)

Bastian, T. S., Bookbinder, J., Dulk, G. A., \& Davis, M. 1990, ApJ, 353, 265

Benz, A. O. 1986, Sol. Phys., 104, 99

Chargeishvili, B., Zhao, J., \& Sakai, J. I. 1993, Sol. Phys., 145, 297

Dulk, G., \& McLean, D. J. 1977, Sol. Phys., 57, 279

Fleishman, G. D., \& Melnikov, V. F. 1998, Adv. Phys. Sci., 168, 1265

Frank, A. G., \& Bogdanov, S. Yu. 2001, EP\&S, 53, 531

Gershberg, R. E. 2002, Activity of Main Sequence Solar Type Stars, Odessa, Astroprint

Gershberg, R. E., \& Pikelner, S. B. 1972, Comments Astrophys. Space Phys., 4, 113

Ginzburg, V. L. 1970, The Propagation of Electromagnetic Waves in Plasmas (Oxford: Pergamon Press)

Ginzburg, V. L., \& Rukhadze, A. A. 1975, Waves in Magnetoactive Plasma (M. Nauka)

Kliem, B., Karlicky, M., \& Benz, A. O. 2000, A\&A, 360, 715
Krucker, S., \& Benz, A. O. 1994, A\&A, 285, 1038

Ledenev, V. G., \& Messerotti, M. 1999, Sol. Phys., 185, 143

Ledenev, V. G., Karlicky, M., Yan, Y., \& Fu, Q. 2001, Sol. Phys., 202, 71

Ledenev, V. G., Tirsky, V. V., \& Tomozov, V. M. 2006, A\&A, 452, 339

Litvinenko, Yu. E. 1996, ApJ, 462, 997

Litvinenko, Yu. E. 2002, Sol. Phys., 212, 379

Mann, G., Karlicky, M., \& Motschmann, U. 1987, Sol. Phys., 110, 381

Melrose, D. B. 1980, Plasma Astrophysics (N-Y: Gordon and Breach)

Mikhailovsky, A.B. 1974, Theory of Plasma Instabilities, Consultant Bureau, N.-Y.

Nakariakov, V. M., Melnikov, V. F., \& Reznikova, V. E. 2003, A\&A, 412, L7

Priest, E. R., \& Forbes, T. 2000, Magnetic reconnection, MHD theory and applications (Cambridge University Press)

Roberts, B., Edvin, P. M., \& Benz, A. O. 1983, Nature, 305, 688

Sakai, J. I., \& de Jager, C. 1996, Space Sci. Rev., 77, 1

Somov, B. V. 1992, Physical processes in solar flares (Dordrecht: Kluwer Academic Publ.)

Stepanov, A.V. 2003, Advances of Physical Sciences, 173, 106

Vrŝnak, B., Magdalenic, J., Aurass, H., \& Mann, G. 2002, A\&A, 396, 673

Zheleznyakov, V. V. 1970, Radio Emission from the Sun and Planets (Oxford: Pergamon Press) 